\title{
High-precision measurements of extensive air showers with the SKA
}

T. Huege ${ }^{* 1}$, J.D. Bray ${ }^{2}$, S. Buitink ${ }^{3}$, R. Dallier ${ }^{4,5}$, R.D. Ekers ${ }^{6}$, H. Falcke ${ }^{7,8}$, A. Haungs ${ }^{1}$, C.W. James ${ }^{9}$, L. Martin ${ }^{4,5}$, B. Revenu ${ }^{4}$, O. Scholten ${ }^{10,11}$, F.G. Schröder ${ }^{1}$ and A. Zilles ${ }^{12}$

${ }^{1}$ IKP, Karlsruher Institut für Technologie, Postfach 3640, 76021 Karlsruhe, Germany

${ }^{2}$ School of Physics \& Astronomy, Univ. of Manchester, M13 9PL, United Kingdom

${ }^{3}$ Astrophysical Institute, Vrije Universiteit Brussel, Pleinlaan 2, 1050 Brussels, Belgium

${ }^{4}$ Subatech, 4 rue Alfred Kastler, 44307 Nantes cedex 3, France

${ }^{5}$ Station de radioastronomie de Nançay, Observatoire de Paris, CNRS/INSU, Nançay, France

${ }^{6}$ CSIRO Astronomy \& Space Science, NSW 2122, Australia

${ }^{7}$ Dept. of Astrophysics/IMAPP, Radboud Univ. Nijmegen, 6500 GL Nijmegen, The Netherlands

${ }^{8}$ Netherlands Institute for Radio Astronomy (ASTRON), 7990 AA Dwingeloo, The Netherlands

${ }^{9}$ ECAP, Univ. of Erlangen-Nuremberg, 91058 Erlangen, Germany

${ }^{10}$ Kernfysisch Versneller Instituut, Univ. of Groningen, 9747 AA Groningen, The Netherlands

${ }^{11}$ Interuniversity Institute for High-Energy, Vrije Universiteit Brussel, Pleinlaan 2, 1050

Brussels, Belgium

${ }^{12}$ EKP, Karlsruher Institut für Technologie, Kaiserstr. 12, 76131 Karlsruhe, Germany

E-mail: tim.huege@kit.edu

As of 2023, the Square Kilometre Array will constitute the world's largest radio telescope, offering unprecedented capabilities for a diverse science programme in radio astronomy. At the same time, the SKA will be ideally suited to detect extensive air showers initiated by cosmic rays in the Earth's atmosphere via their radio emission. With its very dense and uniform antenna spacing in a fiducial area of one $\mathrm{km}^{2}$ and its large bandwidth of 50-350 MHz, the low-frequency part of the SKA will provide very precise measurements of individual cosmic ray air showers. These precision measurements will allow detailed studies of the mass composition of cosmic rays in the energy region of transition from a Galactic to an extragalactic origin. Also, the SKA will facilitate three-dimensional "tomography" of the electromagnetic cascades of air showers, allowing the study of particle interactions at energies beyond the reach of the LHC. Finally, studies of possible connections between air showers and lightning initiation can be taken to a new level with the SKA. We discuss the science potential of air shower detection with the SKA and report on the technical requirements and project status.

The 34th International Cosmic Ray Conference,

30 July- 6 August, 2015

The Hague, The Netherlands

* Speaker. 


\section{Introduction}

Radio detection of extensive air showers has made great progress in the past decade [1]. Currently, the second generation of digital radio detection experiments is in full swing. Two complementary strategies are being followed. One concept is to instrument large areas, spacing antennas as sparsely as possible, as is done in the Auger Engineering Radio Array (AERA) [2] with 150 antennas spanning an area of $\approx 17 \mathrm{~km}^{2}$. The other approach is to observe air showers with a very dense array of antennas on a fairly small area, as is the case in LOFAR [3], with several hundred antennas on an area of $\approx 0.2 \mathrm{~km}^{2}$.

With the latter approach, very detailed information on individual air showers can be gathered. Matching state-of-the-art simulations of the radio signal from extensive air showers [4] to measured LOFAR data allows in particular an accurate reconstruction of the depth of shower maximum $\left(X_{\max }\right)$ of extensive air showers. The average $X_{\max }$ uncertainty of LOFAR measurements has been quantified at $17 \mathrm{~g} / \mathrm{cm}^{2}[5]$.

As of 2020, the low-frequency part of the first phase of the Square Kilometre Array (SKAlow) will go into operation in Australia, its completion being envisaged for 2023. Already in this first phase, the core of SKA-low will be comprised of approximately 60,000 dual-polarized antennas deployed in a circle with $750 \mathrm{~m}$ diameter. With moderate engineering changes to the SKA-low baseline design, these antennas can be used for high-precision measurements of extensive air showers, in a fiducial area of roughly one $\mathrm{km}^{2}$. The SKA focus group on high-energy cosmic particles ${ }^{1}$ has undertaken the effort to work with the SKA organization towards enabling cosmic ray detection with the SKA using extensive air showers (this article) as well as lunar detection [6].

In this article, we shortly discuss the science that could be carried out with SKA-low ${ }^{2}$ once enabled for air-shower detection. Then, we discuss the needed engineering changes to enable SKAlow as a cosmic-ray detector. Finally, we provide a glimpse at the potential of air shower detection with SKA-low on the basis of simulation studies.

\section{Science potential}

The key point of the radio detection of extensive air showers with the SKA will be precision. The high number of antennas measuring individual air showers, their homogeneous distribution in the SKA-low core and the large instantaneous bandwidth from 50 to $350 \mathrm{MHz}$ will allow measurements with unprecedented quality. Measurements of $\sim 10,000$ showers above an energy of $10^{17} \mathrm{eV}$ are expected per year. With these high-fidelity data, the following scientific questions can be addressed:

\subsection{Transition from Galactic to extragalactic cosmic rays}

With SKA-low measurements the mass composition in the energy range from $\gtrsim 10^{16} \mathrm{eV}$ up to $\gtrsim 10^{18} \mathrm{eV}$ can be probed with unprecedented resolution. This could in particular shed light on the transition from Galactic to extragalactic cosmic rays. With LOFAR, the depth of shower

\footnotetext{
${ }^{1}$ http://astronomers.skatelescope.org/home/focus-groups/high-energy-cosmic-particles/

${ }^{2}$ Throughout this article, we use "SKA-low" to refer to the Phase 1 instrument only. Phase 2 is expected to be completed by 2030 and anticipated to be at least four times the size of Phase 1.
} 

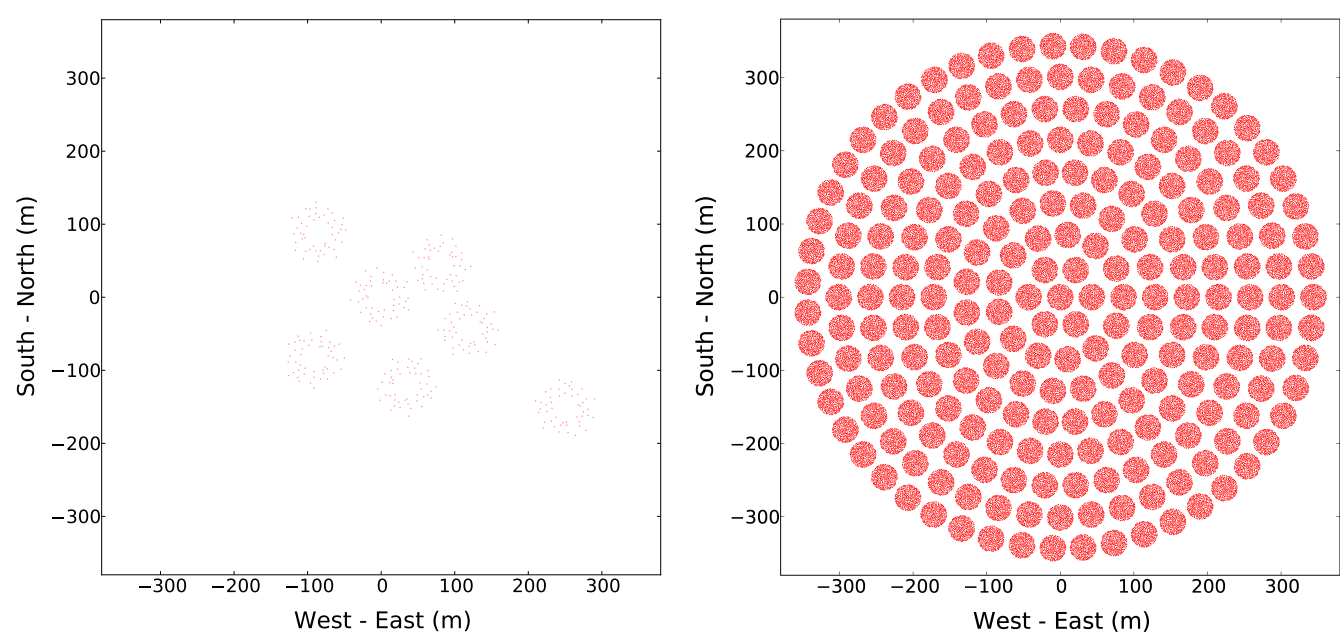

Figure 1: Illustrations of the antenna distributions typically used for air shower observations in the LOFAR core (left) and the SKA-low core (right). Each point represents one dual-polarized antenna. The SKA-low is organized in stations with a diameter of $35 \mathrm{~m}$ comprised of 256 antennas each.

maximum has been successfully reconstructed with a mean accuracy of $17 \mathrm{~g} / \mathrm{cm}^{2}$. This resolution is limited by the incomplete sampling of the radio emission footprint with the inhomogeneous antenna distribution of LOFAR, see Fig. 1 (left). In the case of individual air shower events for which the important features in the radio emission footprint (lateral slope, asymmetry, Cherenkov bump) are well-sampled in spite of the inhomogeneous distribution of antennas LOFAR can achieve a precision on the depth of shower maximum as low as $8 \mathrm{~g} / \mathrm{cm}^{2}$. In comparison, SKA-low will provide much more detailed and much more homogeneous measurements of the radio emission footprint, as is illustrated in Fig. 1 (right). With this and other improvements, we expect to achieve a mean $X_{\max }$ resolution below $10 \mathrm{~g} / \mathrm{cm}^{2}$, significantly better than the $X_{\max }$ resolution of any other technique available to date. Also, these high-precision measurements will be carried out with a near-100\% duty cycle. Identification of individual elements in the cosmic ray flux, in particular the separation between proton showers and other nuclei, will thus become feasible.

\subsection{Air shower physics beyond the LHC scale}

Uncertainties in particle interactions at energies beyond those reached by the LHC and in the regime of extreme forward kinematics still limit the interpretation of air shower data in many ways. Measurements of the moments of the $X_{\max }$ distributions can be used to probe high-energy particle interactions, in particular with respect to the proton-air cross section, secondary particle multiplicity, elasticity and pion charge ratio [7]. With its very accurate $X_{\max }$ determination, a much cleaner separation between proton and helium nuclei than previously achieved should be feasible. SKA-low in particular has the potential to perform measurements of the proton-air cross section [8] with small systematic uncertainties.

Radio signals from extensive air showers, however, contain much more information than just the depth of shower maximum. The entire longitudinal evolution of the air shower is encoded in the radio signal. Using near-field interferometric analysis techniques, it should be possible to perform a three-dimensional "tomography" of the electromagnetic cascade of extensive air showers, 
potentially allowing very detailed studies of air-shower physics. The large number of antennas, their homogeneous spacing and the large instantaneous bandwidth reaching up to $350 \mathrm{MHz}$ will be key to realizing this approach.

\subsection{Cosmic rays and thunderstorms}

Already with LOPES it has been demonstrated that atmospheric electric fields during thunderstorms influence radio emission from extensive air showers [9]. With LOFAR measurements, it was recently demonstrated that the radio detection of extensive air showers during thunderstorm conditions can probe the atmospheric electric fields in thunderclouds [10]. Such measurements provide the unique possibility of studying the conditions leading to lightning initiation in situ. SKA-low will take such measurements to the next level, allowing very detailed studies of thundercloud systems. It has also been hypothesized that the ionisation introduced by extensive air showers could be at the heart of lightning initiation, e.g., via the so-called "relativistic runaway breakdown" mechanism [11], or by providing seed electrons that initiate discharges in the enhanced fields near hydrometeors [12]. With simultaneous measurements of radio pulses emitted by lightning strikes and the radio emission from coincident extensive air showers, SKA-low could also shed new light on this question.

\subsection{Precision studies of air shower radio emission}

Finally, the high-fidelity data of SKA-low can be used to study the radio emission from extensive air showers itself with unprecedented precision. This way it will be possible to validate state-of-the-art simulation codes $[4,13]$ at an extreme level of detail, and at much higher frequencies than possible in experiments existing so far. As these simulation codes are used widely as the basis for design studies and event reconstruction strategies, the scientific community would profit enormously from a high-quality validation of the calculations.

\section{Engineering changes}

SKA-low was originally foreseen as an observatory with its baseline design optimized to various radio-astronomical observation modes. The baseline design is thus not suited to perform measurements of extensive air showers. Here, we discuss the engineering changes that are needed to enable SKA-low as a detector for extensive air showers.

\subsection{Buffering of radio data}

SKA-low is an aperture-synthesis array with individual radio antennas sensitive to the full sky. The signals measured by individual antennas are transmitted to a central location via analog, optical "RF over fibre" links. There, they are digitized and processed further to form beams in certain directions on the sky as governed by the ongoing astronomical observations.

As extensive air showers arrive randomly on the sky, without a priori knowledge of direction and arrival time, air shower detection needs access to the non-beamformed data of the individual antennas. The individual antenna signals thus need to be buffered for long enough until a trigger marking the arrival of an extensive air shower has been received and initiates the buffer readout. 
(We foresee a particle detector array to deliver such a trigger within $10 \mathrm{~ms}$, see next section.) The most important engineering change to SKA-low is thus the installation of suitable buffering capacity at individual antennas. These need to store the raw, unprocessed signal for each polarization of each antenna, with a dynamic range of at least 8 bits, ideally 12 bits. The sampling rate of SKA-low is 800 MSPS. Buffering for air shower detection should be completely independent of other buffering schemes as, e.g., foreseen for the detection of astronomical transients, and should be working continuously, independent of other ongoing observations. In this way, radio detection will be available with a near $100 \%$ duty-cycle, and will be completely commensal to astronomical observations with the SKA.

\subsection{Triggering with particle detectors}

The most reliable way to provide an efficient and pure trigger for the readout of the antenna buffers is a particle detector array. Radio-only triggering would require very powerful real-time analysis of the SKA-low data, and would yield a higher detection threshold and probably a high rate of false positive triggers.

The particle detector should become efficient at $\gtrsim 10^{16} \mathrm{eV}$ and deliver a trigger within $10 \mathrm{~ms}$. Its main purpose is to provide a reliable trigger for the readout of the radio antennas. The reconstruction of the cosmic ray parameters, in particular geometry, energy, and depth of shower maximum, will rely mostly on the high-fidelity radio measurements. A certain separation in the measurement of the electromagnetic and muonic component of the particle cascade will be possible by exploiting the timing and the spatial distribution of the signals measured with the particle detectors. Possibly, a subset of the particle detectors could also be buried to shield the electromagnetic component of the cascade.

One option for the installation of such a particle detector array is to re-use scintillation detectors from the now-dismantled KASCADE array [14]. A total of 180 high-quality scintillator stations consisting of 4 tiles each with dimensions of $90 \times 90 \times 3 \mathrm{~cm}^{3}$ could be used to instrument a circular area with a diameter of $1.1 \mathrm{~km}$, as is shown in Fig. 2. Air showers impacting within this fiducial area can be reconstructed, even if their core is outside the SKA-low antenna core.

Particular care has to be taken not to introduce any radio-frequency interference in SKA-low via the particle detectors, as they will be deployed in very close proximity to the radio antennas. Thorough shielding of the detectors, and the use of silicon photomultipliers instead of photomultiplier tubes operated at high voltage, will ensure that no impulsive radio signals emanate from the detectors. Furthermore, data readout will be performed with the same "RF over fibre" optical links as used for antennas, i.e., digitization and coincidence searches will take place in a central location, and no clock needs to be distributed to the particle detectors. The only electrical connection will be for power.

The particle detector signals will have to be monitored continuously for coincidences and a sophisticated trigger logic will have to be implemented. The goal is to achieve detection unbiased by primary particle type at energies $\gtrsim 10^{16} \mathrm{eV}$, yet keep the event rate to at most one per minute so as to not put too big a strain on the bandwidth needed to read out the buffers. On a trigger being received, the buffers will be frozen to read out a window of $50 \mu \mathrm{s}$ or less, and the data analysed offline. 


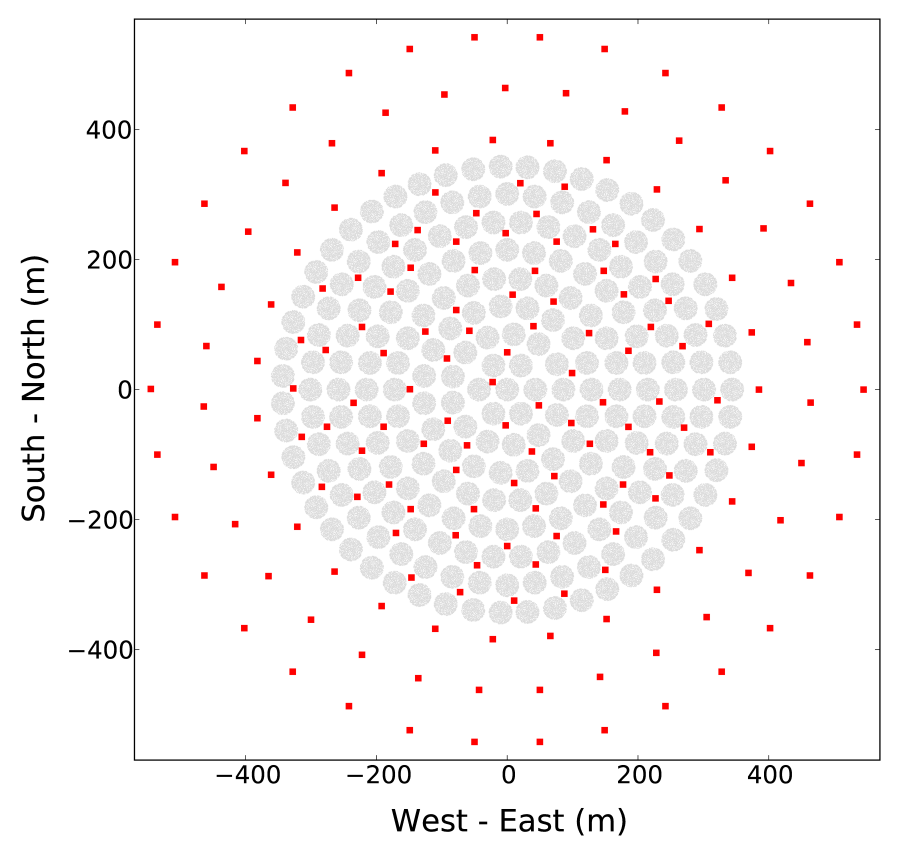

Figure 2: Possible layout of the particle detector array. Red squares: 180 particle detector units, gray circles: 230 SKA-low antenna stations with 256 individual antennas each. The exact layout is still under review.

\section{Initial simulation studies}

We have performed initial simulation studies based on CoREAS [4] simulations to assess the improvement in the measurement of individual air showers with SKA-low as compared to LOFAR. In Fig. 3 we illustrate the amount of detail that a measurement with SKA-low (bottom, zoomed-in at top-right) will record as compared with LOFAR (top-left). The homogeneous and dense sampling of the radio emission in SKA-low will enable reconstruction of the depth of shower maximum with an estimated average uncertainty of less than $10 \mathrm{~g} / \mathrm{cm}^{2}$. The widely-used fluorescence detection technique currently provides depth of shower maximum measurements with a resolution of $\sim 20 \mathrm{~g} / \mathrm{cm}^{2}$, with a duty-cycle of roughly $10 \%$. SKA-low can thus be expected to provide significantly improved resolution of the depth of shower maximum, including systematic uncertainties, and with near-100\% duty-cycle. The high-frequency data available in SKA-low measurements will in addition provide further information such as the diameter of the Cherenkov ring and a measurement of inhomogeneities in the shower cascade.

\section{Conclusion}

With moderate engineering changes, the Square Kilometre Array can be enabled for the detection of extensive air showers. Its approximately 60,000 antennas in a fiducial area of roughly $1 \mathrm{~km}^{2}$ will allow studies of individual air showers at an unprecedented level of detail. We expect an average uncertainty on the determination of the depth of shower maximum of less than $10 \mathrm{~g} / \mathrm{cm}^{2}$, significantly lower than the resolution of the widely-used fluorescence technique, and additionally information on fine details of the air shower longitudinal profile. Along with a precise determi- 

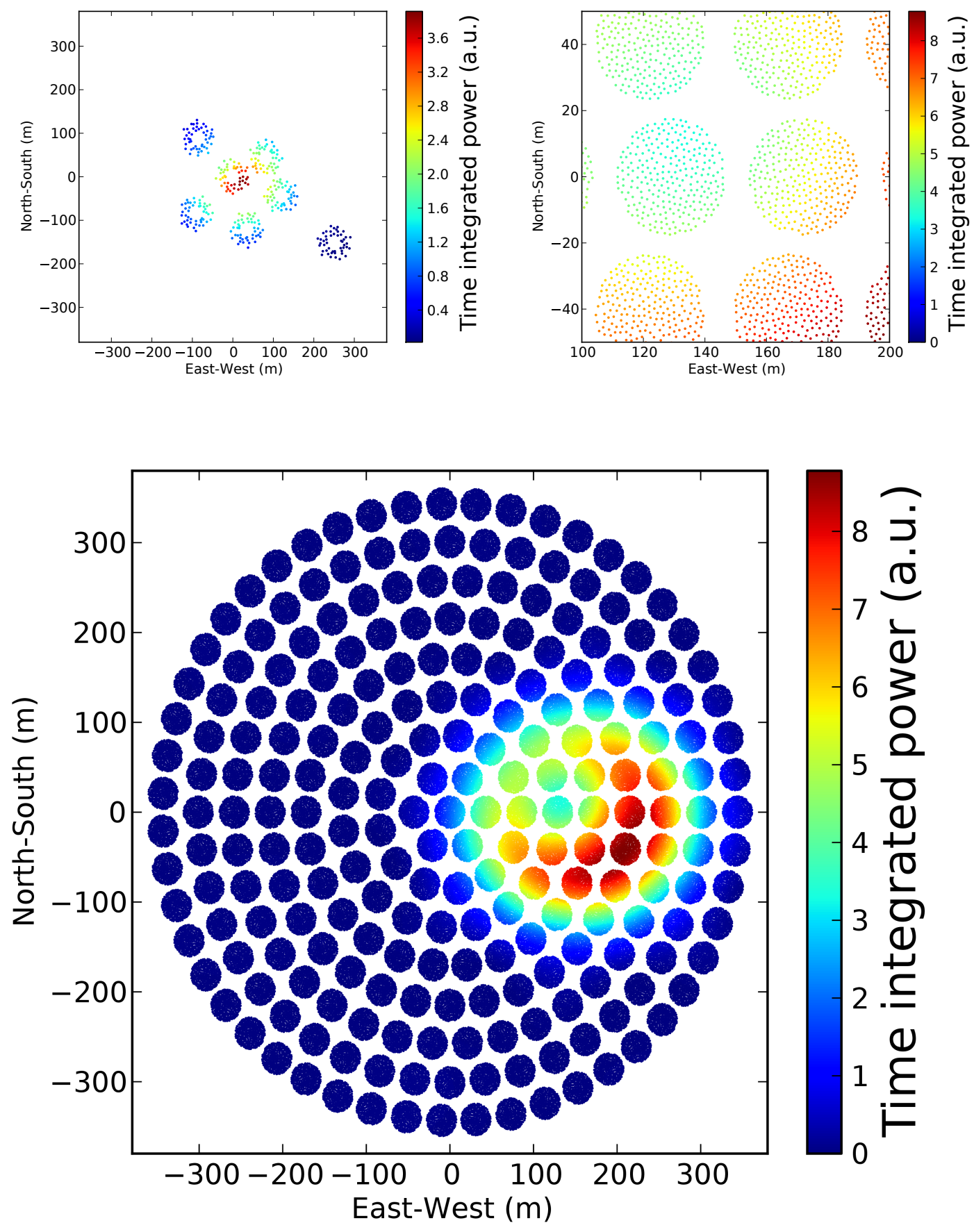

Figure 3: CoREAS simulation of the radio emission footprint sampled with LOFAR (top-left) and SKAlow (bottom, zoomed-in at top-right). Each point corresponds to a measurement with an individual dualpolarized antenna. The zenith angle of the air shower is $30^{\circ}$ and the energy corresponds to $10^{18} \mathrm{eV}$. Even for an ideal core position of the air shower, the LOFAR measurement provides only a very incomplete sampling of the radio signal. In comparison, the SKA-low sampling is extremely homogeneous and detailed, irrespective of the core position within the antenna array. Also note the appearance of a Cherenkov ring in the SKA-low measurement, which is due to the measurement of higher-frequency components up to $350 \mathrm{MHz}$. 
nation of the cosmic ray energy, SKA-low will thus be a powerful instrument to study the mass composition in the energy range from $\gtrsim 10^{16}$ to $\gtrsim 10^{18} \mathrm{eV}$. Among its science goals will be the study of the transition from Galactic to extragalactic cosmic rays, particle interactions at energies beyond the reach of the LHC, and studies of thunderstorm physics, including potential links to cosmic rays.

\section{References}

[1] T. Huege: Braz. J. Phys. 44 (2014) 520

[2] J. Schulz for the Pierre Auger Coll.: this issue (2015), id \#615

[3] P. Schellart, et al.: Astron. Astroph. 560 (2013) A98

[4] T. Huege, M. Ludwig \& C.W. James, AIP Conf. Proc. 1535 (2012) 128

[5] S. Buitink, et al., Phys. Rev. D. 90 (2014) 082003

[6] C.W. James, et al: this issue (2015) id \#291

[7] R. Ulrich, et al., Phys. Rev. D 83 (2011) 054026

[8] P. Abreu, et al. (Pierre Auger Coll.), Phys. Rev. Lett. 109 (2012) 062002

[9] S. Buitink, et al. (LOPES Coll.), A\&A 467 (2007) 385

[10] P. Schellart, et al: Phys. Rev. Lett. 114 (2015) 165001

[11] A.V. Gurevich \& A.N. Karashtin, Phys. Rev. Lett. 110 (2013) 185005

[12] A. Dubinova, et al., Phys. Rev. Lett. 115 (2015) 015002

[13] J. Alvarez-Muñiz, W.R. Carvalho \& E. Zas, Astropart. Phys. 35 (2012) 325

[14] T. Antoni et al. (KASCADE Coll.): Nucl. Instr. Meth. A 513 (2003) 490 